\title{
Mechanism-Specific Therapy of Ventricular Tachycardia
}

\author{
ALAN KADISH, M.D. and FRED MORADY, M.D. \\ From the Division of Cardiology, Department of Internal Medicine, University of Michigan Medical Center, Ann \\ Arbor, Michigan
}

\begin{abstract}
Arrhythmia Mechanisms and Therapy. An understanding of the mechanisms responsible for experimental and clinical arrhythmias has led to the development of mechanism-specific therapies for ventricular tachycardia. These include: (1) beta blockade for catecholamine-sensitive ventricular tachycardia; (2) verapamil for ventricular tachycardia possibly due to triggered activity; (3) pacing, isoproterenol and magnesium therapy for torsade de pointes possibly due to early afterdepolarizations; (4) catheter ablation of the right bundle for bundle branch reentry tachycardia; and (5) catheter ablation of the zone of silow conduction for ventricular tachycardia due to reentry. Hopefully, further progress will be made in removing some of the empiricism from antiarrhythmic therapy.
\end{abstract}

\section{Introduction}

A large number of important descriptive and mechanistic studies have been performed in patients with ventricular tachyarrhythmias (Wellens, et al., 1972; Josephson, et al., 1978[b]; Mason and Winkle, 1978; Kastor, et al., 1981; Waldo, et al., 1984), and considerable progress has been made in the understanding of the basic ionic and electrophysiological mechanisms of arrhythmogenesis. Although it is reasonable to expect that an understanding of basic electrophysiology will lead to improved therapy for arrhythmias, this expectation has been only partially fulfilled. In only a minority of patients does therapy truly depend on an understanding of the basic mechanism of the arrhythmia. This article will review those situations in which an understanding of the mechanism of ventricular tachycardia has helped either to define therapy or at least to explain why particular therapies are efficacious.

\section{Catecholamine-Sensitive Ventricular Tachycardia}

Physiological or psychological stress can precipitate ventricular tachyarrhythmias (Lown, et

Address for correspondence: Fred Morady, M.D., University of Michigan Hospital, 1500 E. Medical Center Drive, Cardiology, B1-F245, Ann Arbor, MI 48109-0022.

Manuscript received 4 October 1989; Accepted for publication 13 October 1989. al., 1977). Both alpha and beta agonists have a large number of electrophysiological effects that could potentiate arrhythmias by a variety of mechanisms. Beta agonists increase the rate of spontaneous phase 4 diastolic depolarization in Purkinje fibers and therefore could potentiate abnormal automaticity (Kassebaum and VanDyke, 1966). Both alpha and beta agonists can increase the amplitude of afterdepolarizations and cause triggered activity (Mary-Rabine, et al., 1980; Kimura, et al., 1984). Finally beta agonists shorten action potential duration (APD) differential shortening of APD in sinus rhythm or with premature beats could increase the dispersion of repolarization and facilitate the development of reentry (Autenrieth, et al., 1975). Thus therapy for catecholamine-sensitive ventricular tachycardia with adrenergic antagonist may not be truly mechanism-specific, but will be included in this discussion because in selected patients a specific mechanism may be suggested and because a knowledge that the arrhythmia is catecholamine-sensitive may help better define therapy.

Sung et al. described a group of patients in whom ventricular tachycardia occurred during isoproterenol infusion but could not be provoked by programmed stimulation or terminated by overdrive pacing (Sung, et al., 1983). Seven of 11 patients did not have organic heart disease. The characteristics of these tachycardias suggested abnormal automaticity. Beta blockers were effective in abolishing the tachycardia in all 11 patients and 
FIGURE - EIGHT MODEL OF REENTRY

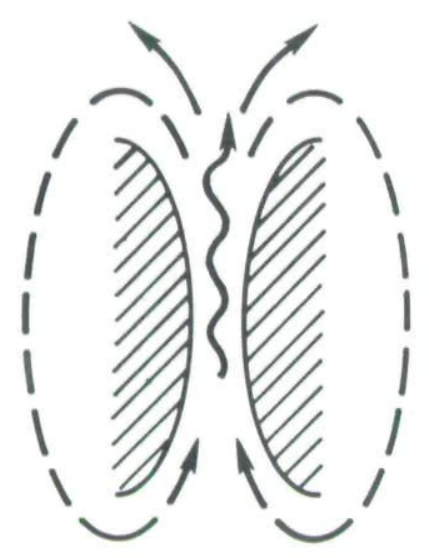

CLASSIC ENTRAINMENT

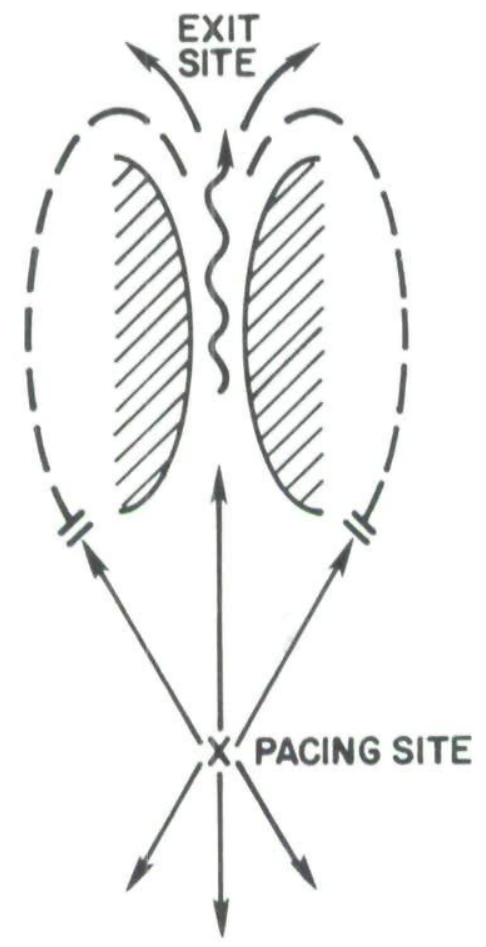

\section{ENTRAINMENT WITH NO CHANGE IN QRS}

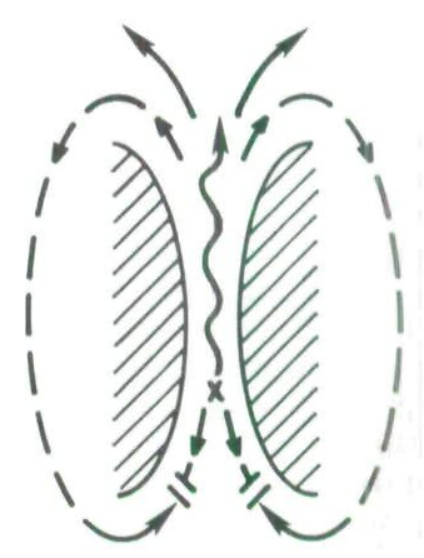

Figure 6. Schematic illustration of the "figure-eight" model of reentry. On the left is shown a zone of slow conduction bounded by 2 arcs of conduction block. The surface QRS begins when the wave of depolarization exits from the "slow zone". During classic entrainment (middle panel), there is fusion between the QRS originating at the pacing site and the QRS resulting from orthodromic conduction through the zone of slow conduction to the exit site. There is a collision of wave fronts in the antidromic direction. "Concealed entrainment, "' or entrainment with no change in the QRS, is depicted in the panel on the right. Because the pacing site is within the zone of slow conduction and bounded by arcs of conduction block, there is no fusion of the QRS. Block in the antidromic direction and slow conduction in the orthodromic direction results in a QRS identical to that of the ventricular tachycardia, with a long-stimulus-to-QRS interval.

\section{References}

Autenrieth G, Surawicz B, Kuo CS, et al: Primary $\mathrm{T}$ wave abnormalities caused by uniform and regional shortening of ventricular monophasic action potential in dog. Circulation 1975;51:668-676.

Bacq ZM, Blakeley AGH, Summers RJ: The effects of amiodarone, an alpha and beta receptor antagonist on adrenergic transmission in the cat spleen. Biochem Pharma 1976;25:1195-1199.

Bailie DS, Inoue H, Kaseda S, et al: Magnesium suppression of early afterdepolarizations and ventricular tachyarrhythmias induced by cesium in dogs. Circulation 1988;77:1395-1402.

Ben-David J, Zipes DP: Differential response to right afterdepolarizations and ventricular tachycardia induced by cesium in the dog. Circulation 1988;78:1241-1250.

Bonatti V, Rolli A, Botti G: Recording of monophasic action potentials of the right ventricle in long QT syndromes complicated by severe ven- tricular arrhythmias. Eur Heart J 1983;4:168-171.

Buxton AE, Josephson ME: Ventricular tachycardia-1983. PACE 1984;7:96-108.

Buxton AE, Waxman HL, Marchlinski FE, et al: Electropharmacology of nonsustained ventricular tachycardia: Effects of class I antiarrhythmic agents, verapamil and propranolol. Am J Cardiol 1984;53:738-744.

Caceres J, Jazayeri M, McKinnie J, et al: Sustained bundle branch reentry as a mechanism of clinical tachycardia. Circulation 1989;79:256-270.

Chen RF, Kushner JA, Toivonen LK, et al: Beta blocking effects of amiodarone. (abstract) Circulation 1988;78(Suppl):II.

Cox JL: Patient selection criteria and results of surgery for refractory ischemic ventricular tachycardia. Circulation 1989;79(Suppl I)I:I-163.

El-Sherif N, Bekheit SS, Henkin R: Quinidineinduced long QTU interval Torsade de Pointes: role of bradycardia-dependent early afterdepolarizations. J Am Coll Cardiol 1989;14:252-257. 